\title{
Isoparametric Curve of Quadratic F-Bézier Curve
}

\author{
Hae Yeon Park ${ }^{1}$ and Young Joon Ahn ${ }^{2, \dagger}$
}

\begin{abstract}
In this thesis, we consider isoparametric curves of quadratic F-Bézier curves. F-Bézier curves unify C-Bézier curves whose basis is $\{\sin t, \cos t, t, 1\}$ and H-Bézier curves whose basis is $\{\sinh t, \cosh t, t, 1\}$. Thus F-Bézier curves are more useful in Geometric Modeling or CAGD(Computer Aided Geometric Design). We derive the relation between the quadratic F-Bézier curves and the quadratic rational Bézier curves. We also obtain the geometric properties of isoparametric curve of the quadratic F-Bézier curves at both end points and prove the continuity of the isoparametric curve.
\end{abstract}

Key words : F-Bézier Curve, Quadratic Rational Bézier Curve, Isoparametric Curve, Q-Bézier Curve, H-Bézier Curve

\section{Introduction}

C-Bézier curve ${ }^{[1,2]}$ is an extension of Beizer curve using $\cos (t)$ and $\sin (t)$, which is used to express circular arc, ellipse, cycloid and helix. Zhang developed the C-B-spline from the C-Bézier curves ${ }^{[3]}$ and C-Bézier curves and surfaces ${ }^{[4]}$. It is more advantage of F-Bézier curve than rational Bézier curve to able to express helix or cycloid $^{[5]}$. Chen and Wang ${ }^{[6]}$ presented C-Bézier curves of higher degree.

H-Bézier curve ${ }^{[7]}$ is the curve combined by Bézier curve and hyperbolic functions $\cosh (t), \sinh (t)$. Zhang and Krause ${ }^{[8]}$ unified these curves into F-Bézier curve. Zhang et al. ${ }^{[9]}$ also unified the coefficient of F-Bézier curves using complex number.

Every quadratic F-Bézier curve has the same graph of a quadratic rational Bézier curve, and vice versa. Any isoparametric curve of quadratic Bézier curves is a straight line, but that of F-Bézier curves is not. So we study the isoparametric curve of quadratic F-Bézier curves in this paper. We show that the isoparametric curve is tangent to the polygon at the end point of the curve, or a straight line. We also prove that the iso-

${ }^{1}$ Graduate School of Education, Chosun University, Gwangju 501-759, Korea

${ }^{2}$ Department of Mathematics Education, Chosun University, Gwangju 501-759, Korea

${ }^{\dagger}$ Corresponding Author : ahn@chosun.ac.kr

(Received : January 2, 2013, Revised : March 20, 2013,

Accepted : March 25, 2013) parametric curves are $C^{1}$ continuous but not twice differentiable.

This paper is constructed as follows. In Section 2, the well-known facts about the quadratic rational Bézier curves and the quadratic F-Bézier curves are given. In Section 3, we present some geometric properties of F-Bézier curves, and we summary them in Section 4.

\section{Prior Works}

In this section we remind the definition and properties of quadratic rational Bézier curve and quadratic F-Beizer curve.

The quadratic rational Bézier curve is well introduced by Farin ${ }^{[10]}$.

Definition 2.1 Quadratic rational Bézier curve is

$$
r(t)=\frac{\sum_{i=0}^{2} w_{i} B_{i}^{2}(t) \boldsymbol{b}_{i}}{\sum_{i=0}^{2} w_{i} B_{i}^{2}(t)}
$$

where $b_{i}, i=0,1,2$, is control point, $w_{i}>0$ is weight, and $B_{i}^{2}(t)=\left(\begin{array}{l}2 \\ i\end{array}\right) t^{i}(1-t)^{2-i}$ is the quadratic Bernstein polynomial. If $w_{0}=w_{2}=1$, the quadratic Bézier curve is called by standard form.

Any quadratic rational Bézier curve $r(t)$ with control points $b_{i}$ and weights $w_{i}(i=0,1,2)$ can be reparametrized 
by standard form with new weights $1, \frac{w_{1}}{\sqrt{w_{0} w_{2}}}, 1$ without change of graph ${ }^{[6]}$. Actually, using the reparametrization

$$
t(s)=\frac{s}{\rho(1-s)+s}, \rho=\sqrt{\frac{w_{2}}{w_{0}}}
$$

new reparametrized quadratic Bézier curve is obtained by

$$
\begin{aligned}
\hat{r}(s) & =r(t(s)) \\
& =\frac{\rho^{2} w_{0} B_{0}^{2}(s) b_{0}+\rho w_{1} B_{1}^{2}(s) b_{1}+w_{2} B_{2}^{2}(s) b_{2}}{\rho^{2} w_{0} B_{0}^{2}(s)+\rho w_{1} B_{1}^{2}(s)+w_{2} B_{2}^{2}(s)}
\end{aligned}
$$

which is a standard quadratic rational Bézier curve. $r(t(s))$ and $\hat{r}(s)$ have the same graph. Thus any quadratic rational Bézier curve can be rewritten by the standard form

$$
r(t)=\frac{B_{0}^{2}(t) b_{0}+w B_{1}^{2}(t) b_{1}+B_{2}^{2}(t) b_{2}}{B_{0}^{2}(t)+w B_{1}^{2}(t)+B_{2}^{2}(t)}
$$

where $w=\frac{w_{1}}{\sqrt{w_{0} w_{2}}}$. It is well known that $w<1$ iff $r$ is ellipse, $w=1$ iff $r$ is parabola, and $w>1$ iff $r$ is hyperbola, as shown in Fig. $1^{[10]}$.

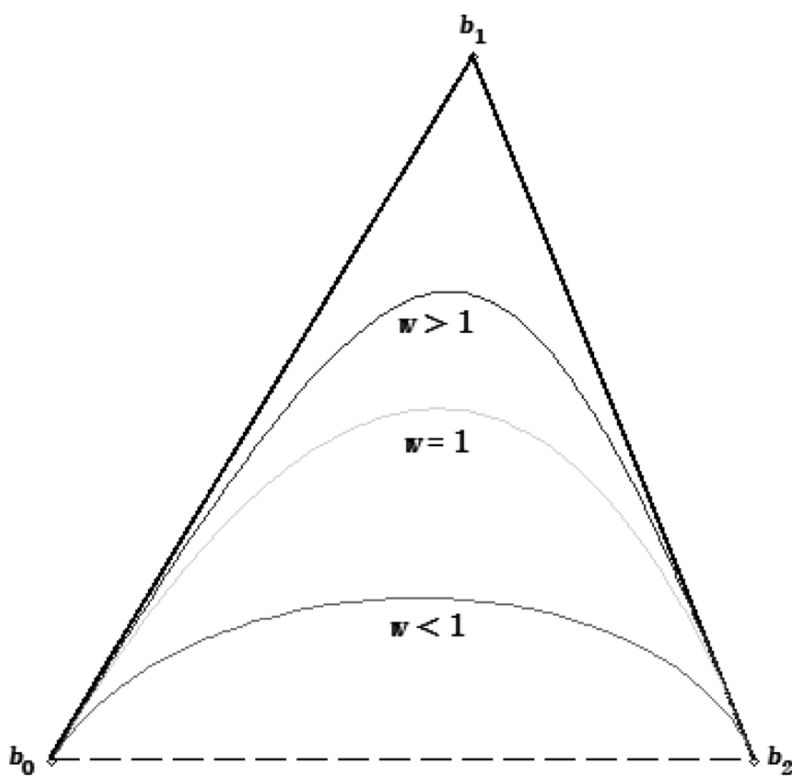

Fig. 1. Quadratic rational Bézier curves with weight $w<1$ (ellipse), $w=1$ (parabola), or $w>1$ (hyperbola). $\left[b_{0}, b_{1}, b_{2}\right]$ is control polygon(black).

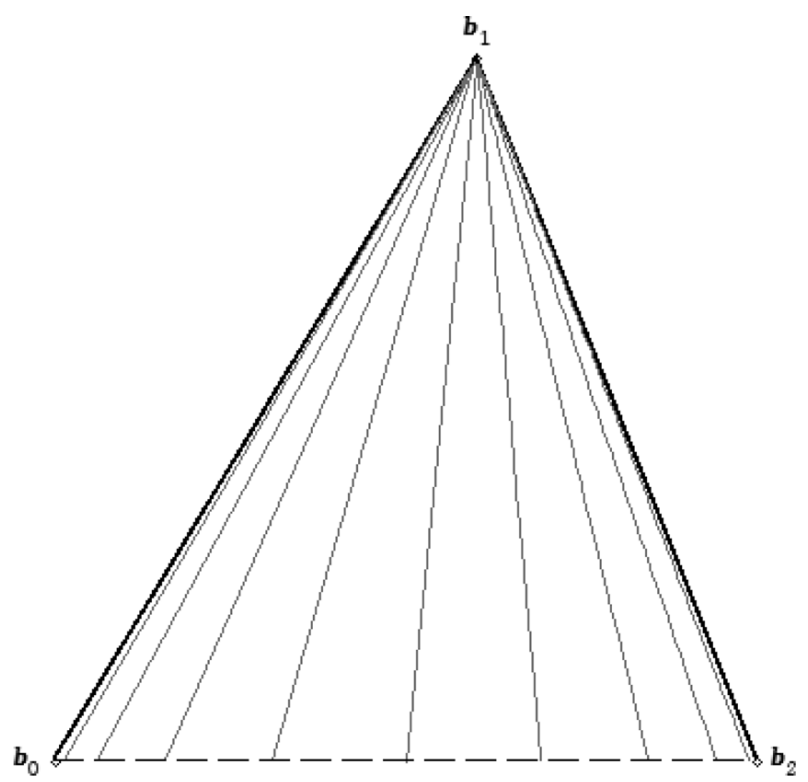

Fig. 2. isoparametric curves of quadratic rational Bézier curves: $r^{*}\left(w, t_{0}\right)$ for $t_{0}=\frac{i}{10},(i=, 1,2, \cdots, 9)$.

It is well known that the function $r^{*}:(0,1) \times(0, \infty)$ $\rightarrow \triangle p_{0} p_{1} p_{2}$ defined by $r^{*}(w, t)=r(t)$ is homeomorphic on $(0,1) \times(0, \infty)$. For each $t_{0} \in(0,1)$, the isoparametric curve is

$$
r^{*}\left(w, t_{0}\right)=b_{1}+\frac{B_{0}^{2}\left(t_{0}\right)\left(b_{0}-b_{1}\right)+B_{2}^{2}\left(t_{0}\right)\left(b_{2}-b_{1}\right)}{B_{0}^{2}\left(t_{0}\right)+w B_{1}^{2}\left(t_{0}\right)+B_{2}^{2}\left(t_{0}\right)}
$$

Hence $r^{*}\left(w, t_{0}\right)$ is a straight line between the two points $\frac{B_{0}^{2}(t) b_{0}+B_{2}^{2}(t) b_{2}}{B_{0}^{2}(t)+B_{2}^{2}(t)}$ and $b_{1}$ as shown in Fig. 2 .

In what follows, we will need to consider the implicit form of a conic section. Any point $(x, y) \in \mathbb{R}^{2}$ can be written uniquely in terms of barycentric coordinates $\left(\tau_{0}, \tau_{1}, \tau_{2}\right)$, where $\tau_{0}+\tau_{1}+\tau_{2}=1$, with respect to the triangle $\triangle p_{0} p_{1} p_{2}:(x, y)=\tau_{0} p_{0}+\tau_{1} p_{1}+\tau_{2} p_{2}$. Consequently any function $f: \triangle b_{0} b_{1} b_{2} \rightarrow \mathbb{R}$ can be expressed as a function of $\tau_{0}, \tau_{1}, \tau_{2}$. The following lemma is well known $^{[10,11]}$.

Lemma 2.2 Let $r$ be a quadratic rational Bézier curve with control points $b_{0}, b_{1}, b_{2}$ and weight $w$ and let $f$ be defined as

$$
f(x, y)=\tau_{1}^{2}-4 w^{2} \tau_{0} \tau_{2}
$$


Then $r$ satisfes the equation $f(r(t))=0$ for all $t \in[0,1]$.

Zhang and Krause ${ }^{[9]}$ unified the C-Bézier curve and H-Bézier curve, which is called F-Bézier curve.

Definition 2.3 The F-Bézier curve of degree $n$ with a parameter $\alpha \in(-\pi, \infty)$ is

$$
q(t)=\sum_{i=0}^{n} Z_{i}^{n}(t) q_{i}
$$

where $q_{i}, i=0,1, \cdots, n$, is control point, and $Z_{i}^{n}$ is the F-basis functions defined by

$$
\begin{aligned}
& Z_{1}^{1}(t)= \begin{cases}\frac{\sin (\alpha t)}{\sin (\alpha)} & (-\pi<\alpha<0) \\
t & (\alpha=0) \\
\frac{\sinh (\alpha t)}{\sinh (\alpha)} & (\alpha>0)\end{cases} \\
& Z_{0}^{1}(t)=Z_{1}^{1}(1-t)
\end{aligned}
$$

and recursively

$$
\begin{aligned}
& W_{i}^{n}(t)=\alpha \int_{0}^{t} Z_{i}^{n}(t) d t \\
& (0 \leqq i \leqq n, n \geqq 1) \\
& Z_{n}^{n}(t)=\frac{W_{n-1}^{n-1}(t)}{W_{n-1}^{n-1}(1)} \\
& Z_{0}^{n}(t)=1-\frac{W_{0}^{n-1}(t)}{W_{0}^{n-1}(1)} \\
& Z_{i}^{n}(t)=\frac{W_{i-1}^{n-1}(t)}{W_{i-1}^{n-1}(1)}-\frac{W_{i}^{n-1}(t)}{W_{i}^{n-1}(1)} \\
& (1 \leqq i \leqq n-1, n \geqq 2) .
\end{aligned}
$$

In this paper we concentrate the quadratic F-Bézier curve, which can be rewritten as follows.

Definition 2.4 The quadratic F-Bézier curve is

$$
q(t)=\sum_{i=0}^{2} Z_{i}^{2}(t) q_{i}
$$

where $q_{i}, i=0,1,2$, is control point, and $Z_{i}^{2}$ is quadratic F-basis functions defined by

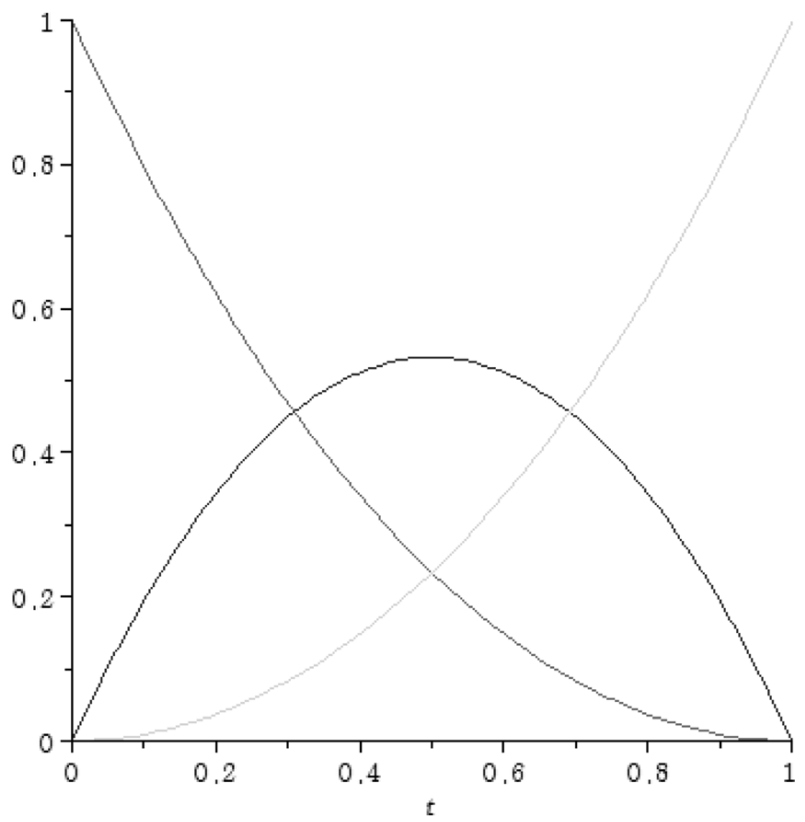

Fig. 3. Basis functions of quadratic F-Bézier curve for $\alpha=\pi / 3: Z_{0}^{2}(t)\left(\right.$ red), $Z_{1}^{2}(t)$ (blue), $Z_{2}^{2}(t)$ (green).

$$
Z_{2}^{2}(t)= \begin{cases}\frac{1-\cos (\alpha t)}{1-\cos \alpha} & (-\pi<\alpha<0) \\ t^{2} & (\alpha=0) \\ \frac{1-\cosh (\alpha t)}{1-\cosh \alpha} & (\alpha>0)\end{cases}
$$

and

$$
\begin{aligned}
& Z_{0}^{2}(t)=Z_{2}^{2}(1-t) \\
& Z_{1}^{2}(t)=1-Z_{0}^{2}(t)-Z_{2}^{2}(t) .
\end{aligned}
$$

As shown in Fig. 3, the basis functions $Z_{i}^{2}(t), i=0,1,2$ satisfy the partition of unity, so the quadratic F-Bézier curves satisfy convex-hull property.

\section{Relation between F-Bézier Curve and Quadratic Rational Bézier Curve}

In this section we present some geometric properties of the isoparametric curves of the quadratic F-Bézier curves. The results in this section is an improvement of the results in the thesis ${ }^{[12]}$ of the first author in this paper. We consider the nonlinear control polygons of F-Bézier curve and quadratic ratonal Bézier curves

$$
q_{0}, q_{1}, q_{2} \text { and } b_{0}, b_{1}, b_{2} \text {. }
$$


Proposition 3.1 F-Bézier curve has the same graph of quadratic rational Bézier curve if and only if $q_{i}=b_{i}$ $(i=0,1,2)$, and

$$
w= \begin{cases}\cos (\alpha / 2) & (-\pi<\alpha \leq 0) \\ \cosh (\alpha / 2) & (\alpha>0)\end{cases}
$$

proof. By Definition 2.4, $q(t)$ has the barycentric coordinates $\left(Z_{0}^{2}(t), Z_{1}^{2}(t), Z_{2}^{2}(t)\right)$, and by Lemma 2.2, $q(t)$ is a conic section iff $Z_{1}^{2}(t) / 2 \sqrt{Z_{0}^{2}(t) Z_{2}^{2}(t)}$ is a constant which is equal to the weight of the conic.

$$
\begin{aligned}
& \text { For }-\pi<\alpha<0, \\
& \frac{Z_{1}^{2}(t)}{2 \sqrt{Z_{0}^{2}(t) Z_{2}^{2}(t)}} \\
= & \frac{1-\frac{1-\cos (\alpha t)}{1-\cos \alpha}-\frac{1-\cos (\alpha(1-t))}{1-\cos \alpha}}{2 \sqrt{\frac{1-\cos (\alpha t)}{1-\cos \alpha} \cdot \frac{1-\cos (\alpha(1-t))}{1-\cos \alpha}}} \\
= & \frac{\cos (\alpha t)-\cos \alpha+\cos (\alpha(1-t))-1}{2 \sqrt{(1-\cos (\alpha t)) \cdot(1-\cos (\alpha(1-t)))}} \\
= & -\frac{1}{2} \frac{\sin \left(\frac{\alpha t+\alpha}{2}\right) \sin \left(\frac{\alpha t-\alpha}{2}\right)+\sin ^{2}\left(\frac{\alpha(1-t)}{2}\right)}{\sin \frac{\alpha t}{2} \cdot \sin \frac{\alpha(1-t)}{2}} \\
= & \frac{1}{2} \frac{\sin \left(\frac{\alpha t+\alpha}{2}\right)-\sin \left(\frac{\alpha(1-t)}{2}\right)}{\sin \left(\frac{\alpha t}{2}\right)} \\
= & \frac{1}{2} \frac{2 \cos \left(\frac{\alpha}{2}\right) \sin \left(\frac{\alpha t}{2}\right)}{\sin \left(\frac{\alpha t}{2}\right)}=\cos \left(\frac{\alpha}{2}\right) .
\end{aligned}
$$

For $\alpha=0$, trivially $Z_{1}^{2}(t) / 2 \sqrt{Z_{0}^{2}(t) Z_{2}^{2}(t)}=1$.

For $\alpha>0$, by the identity $\cosh (x)=\cos (i x)$,

$\frac{Z_{1,2}(t)}{2 \sqrt{Z_{0,2}(t) Z_{2.2}(t)}}$

$=\frac{\cosh (\alpha t)-\cosh \alpha+\cosh (\alpha(1-t))-1}{2 \sqrt{(1-\cosh (\alpha t)) \cdot(1-\cosh (\alpha(1-t)))}}$

$=\cosh \left(\frac{\alpha}{2}\right)$.

Comllary 3.2 If $q_{i}=b_{i}(i=0,1,2)$, and

$$
w= \begin{cases}\cos (\alpha / 2) & (-\pi<\alpha \leq 0) \\ \cosh (\alpha / 2) & (\alpha>0),\end{cases}
$$

then for all $t \in[0,1]$

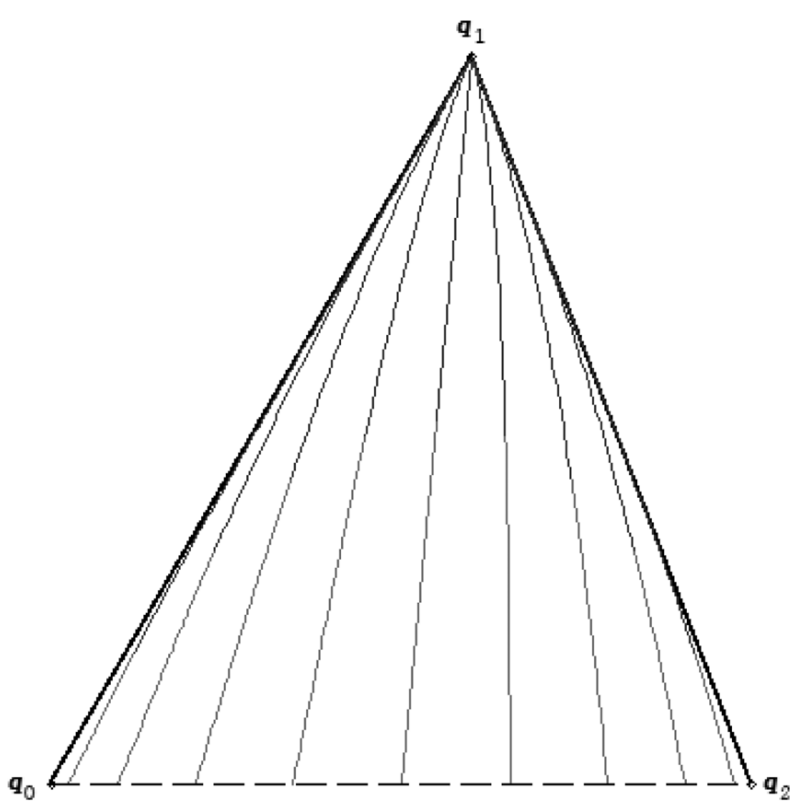

Fig. 4. Isoparametric curves of quadratic F-Bézier curves: $q^{*}\left(\alpha, t_{0}\right)$ for $t_{0}=\frac{i}{10},(i=, 1,2, \cdots, 9)$, for $-\pi<\alpha<0$ (red) and $0<\alpha<\infty$ (blue).

$$
r(t)=\left\{\begin{array}{l}
q\left(-\arccos \left(1-\mu_{2}(t)(1-\cos \alpha)\right) / \alpha\right) \\
q\left(\operatorname{arccosh}\left(1-\mu_{2}(t)(1-\cosh \alpha)\right) / \alpha\right),
\end{array}\right.
$$

respectively, where

$$
\mu_{2}(t)=\frac{B_{2}^{2}(t)}{B_{0}^{2}(t)+w B_{1}^{2}(t)+B_{2}^{2}(t)} .
$$

Actually there are two parameters $\alpha \in(-\pi, \infty)$ and $t \in[0,1]$ in the F-Bézier curve $q(t)$. Let the isoparametric curve $q\left(t_{0}\right)$ with $\alpha \in(-\pi, \infty)$ denoted by $q^{*}\left(\alpha, t_{0}\right)$, as shown Fig. 4. If $t_{0}=0$ or 1 , the the isoparametric curve $q^{*}\left(\alpha, t_{0}\right)$ is a one point $q_{0}$ or $q_{2}$.

Proposition 3.3 For each $t_{0} \in(0,1)$, the isoparametric curve $q^{*}\left(\alpha, t_{0}\right)$ starts at $q^{*}\left(-\pi, t_{0}\right)=\frac{1}{2}\left(\left(1+\cos \left(\pi t_{0}\right)\right)\right.$ $\left.q_{0}+\left(1-\cos \left(\pi t_{0}\right)\right) q_{2}\right)$ and ends at $q_{1}$.

proof. By Definition 2.4,

$$
\begin{aligned}
\lim _{\alpha \rightarrow-\pi} Z_{2}^{2}(t) & =\frac{1-\cos (\pi t)}{2} \\
\lim _{\alpha \rightarrow-\pi} Z_{0}^{2}(t) & =\frac{1+\cos (\pi t)}{2} \\
\lim _{\alpha \rightarrow-\pi} Z_{1}^{2}(t) & =0
\end{aligned}
$$




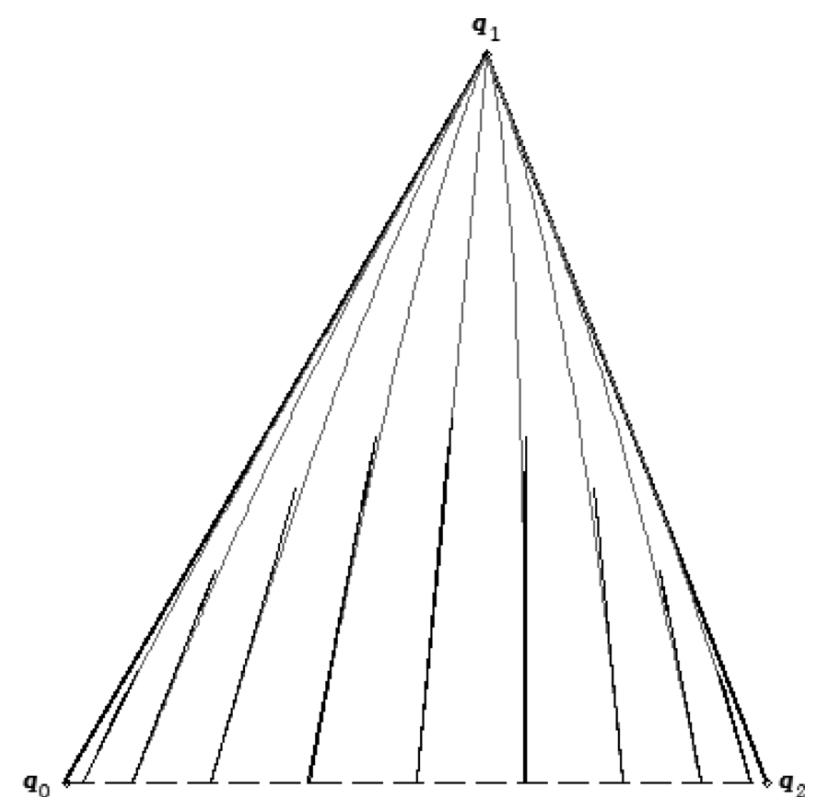

Fig. 5. Tangent vectors(blue) of isoparametric curves(red) of F-Bézier curves $q^{*}\left(\alpha, t_{0}\right)$ at $\alpha=-\pi$.

so the start point is

$q^{*}\left(-\pi, t_{0}\right)=\frac{\left(1+\cos \left(\pi t_{0}\right)\right) q_{0}+\left(1-\cos \left(\pi t_{0}\right)\right) q_{2}}{2}$

and by

$\lim _{\alpha \rightarrow \infty} Z_{2}^{2}(t)=\lim _{\alpha \rightarrow \infty} \frac{1-\cosh (\alpha t)}{1-\cosh \alpha}=0$

$\lim _{\alpha \rightarrow-\pi} Z_{0}^{2}(t)=\lim _{\alpha \rightarrow \infty} \frac{1-\cosh (\alpha(1-t))}{1-\cosh \alpha}=0$

$\lim _{\alpha \rightarrow-\pi} Z_{1}^{2}(t)=1$

the end point is $\lim _{\alpha \rightarrow \infty} q^{*}\left(\alpha, t_{0}\right)=q_{1}$.

Let $D_{\alpha}$ be the partial derivative with respect to $\alpha$, and let $Z_{i}^{*}(\alpha, t)=Z_{i}^{2}(t)$ for $i=0,1,2$.

Proposition 3.4 For each $t_{0} \in(0,1)$, the isoparametric curve $q^{*}\left(\alpha, t_{0}\right)$ is of tangent direction $\frac{1}{2} \sin \left(\pi t_{0}\right)$ $\left(q_{1}-\left(1-t_{0}\right) q_{0}-t_{0} q_{2}\right)$ at the start point $q^{*}\left(-\pi, t_{0}\right)$.

proof. Since for $\alpha \in(-\pi, 0)$

$D_{\alpha} Z_{2}^{*}(\alpha, t)=\frac{\sin (\alpha t) t}{1-\cos \alpha}-\frac{(1-\cos (\alpha t)) \sin \alpha}{(1-\cos \alpha)^{2}}$

we have

$\lim _{\alpha \rightarrow-\pi} D_{\alpha} Z_{2}^{*}(\alpha, t)=-\frac{\sin (\pi t) t}{2}$ and so

$\lim _{\alpha \rightarrow-\pi} D_{\alpha} Z_{0}^{*}(\alpha, t)=-\frac{\sin (\pi t)(1-t)}{2}$

$\lim _{\alpha \rightarrow-\pi} D_{\alpha} Z_{1}^{*}(\alpha, t)=\frac{\sin (\pi t)}{2}$.

By Definition 2.4,

$D_{\alpha} q^{*}\left(-\pi, t_{0}\right)=\frac{\sin \left(\pi t_{0}\right)}{2}\left(q_{1}-\left(1-t_{0}\right) q_{0}-t_{0} q_{2}\right)$.

Theorem 3.5 For each $t_{0} \in(0,1)$, the isoparametric curve $q^{*}\left(\alpha, t_{0}\right)$ is tangent to $\overline{q_{0} q_{1}}, \overline{m q_{1}}$, or $\overline{q_{2} q_{1}}$ at $q_{1}$ iff $0<t_{0}<1 / 2, t_{0}=1 / 2$ or $1 / 2<t_{0}<1$, respectively, where $m=\left(q_{0}+q_{2}\right) / 2$. Moreover, $q^{*}(\alpha, 1 / 2), \quad \alpha \in$ $[-\pi, \infty)$ is a line segment $\overline{m q_{1}}$.

proof. For $\alpha>0$, from Definition 2.4,

$D_{\alpha} Z_{2}^{*}(\alpha, t)=-\frac{\sinh (\alpha t) t}{1-\cosh \alpha}+\frac{(1-\cosh (\alpha t)) \sinh \alpha}{(1-\cosh \alpha)^{2}}$

and so

$$
\begin{aligned}
D_{\alpha} Z_{0}^{*}(\alpha, t) & =-\frac{\sinh (\alpha(1-t))(1-t)}{1-\cosh \alpha} \\
& +\frac{(1-\cosh (\alpha(1-t))) \sinh \alpha}{(1-\cosh \alpha)^{2}} \\
D_{\alpha} Z_{1}^{*}(\alpha, t) & =-D_{\alpha} Z_{0}^{*}(\alpha, t)-D_{\alpha} Z_{2}^{*}(\alpha, t) .
\end{aligned}
$$

Thus $\lim _{\alpha \rightarrow \infty} \frac{D_{\alpha} Z_{0}^{*}(\alpha, t)}{D_{\alpha} Z_{2}^{*}(\alpha, t)}=\infty, 1$, or 0 iff $t<\frac{1}{2}$, $t_{0}=\frac{1}{2}$, or $t>\frac{1}{2}$, respectively. If $0<t_{0}<\frac{1}{2}$,

then

$\lim _{t \rightarrow \infty} \frac{D_{\alpha} Z_{1}^{*}(\alpha, t)}{D_{\alpha} Z_{0}^{*}(\alpha, t)}=-1$,

so that

$\lim _{t \rightarrow \infty} \frac{D_{\alpha} q^{*}(\alpha, t)}{D_{\alpha} Z_{0}^{*}(\alpha, t)}$

$=\lim _{t \rightarrow \infty} \frac{D_{\alpha} Z_{0}^{*}(\alpha, t) q_{0}+D_{\alpha} Z_{1}^{*}(\alpha, t) q_{1}+D_{\alpha} Z_{2}^{*}(\alpha, t) q_{2}}{D_{\alpha} Z_{0}^{*}(\alpha, t)}$

$=q_{0}-q_{1}$.

If $1 / 2<t_{0}<1$, then

$\lim _{t \rightarrow \infty} \frac{D_{\alpha} Z_{1}^{*}(\alpha, t)}{D_{\alpha} Z_{2}^{*}(\alpha, t)}=-1$,

so that

$\lim _{t \rightarrow \infty} \frac{D_{\alpha} q^{*}(\alpha, t)}{D_{\alpha} Z_{2}^{*}(\alpha, t)}=q_{2}-q_{1}$. 
If $t_{0}=1 / 2$, then $Z_{0}^{*}(\alpha, 1 / 2)=Z_{2}^{*}(\alpha, 1 / 2)$ and $q^{*}(\alpha, 1 / 2)=2 Z_{0}^{*}(\alpha, 1 / 2) m+\left(1-2 Z_{0}^{*}(\alpha, 1 / 2)\right) \boldsymbol{q}_{1}$.

Since $Z_{0}^{*}(\alpha, 1 / 2) \in(0,1 / 2]$ for $\alpha \in[-\pi, \infty)$, the isoparametric curve $q^{*}(\alpha, 1 / 2)$ is a line segment $\overline{m q_{1}}$.

Thus the isoparametric curve $q^{*}\left(\alpha, t_{0}\right)$ is tangent to $\overline{q_{0} q_{1}}, \overline{m q_{1}}$, or $\overline{q_{1} q_{2}}$ at $q_{1}$ iff $0<t_{0}<1 / 2, t_{0}=1 / 2$ or $1 / 2<t_{0}<1$, respectively.

Proposition 3.6 For each $t_{0} \in(0,1)$, the isoparametric curve $q^{*}\left(\alpha, t_{0}\right), \alpha \in(-\pi, \infty)$ is a $C^{1}$ continuous curve.

proof. It is sufficient to show that $q^{*}\left(\alpha, t_{0}\right)$ is continuous and differentiable at $\alpha=0$. By the series expansion

$$
\begin{aligned}
\frac{1-\cos (\alpha t)}{1-\cos \alpha} & =\frac{\frac{t^{2}}{2 !} \alpha^{2}-\frac{t^{4}}{4 !} \alpha^{4}+\cdots}{\frac{\alpha^{2}}{2 !}-\frac{\alpha^{4}}{4 !}+\cdots}=\frac{t^{2}-\frac{2 t^{4}}{4 !} \alpha^{2}+\cdots}{1-\frac{2 \alpha^{2}}{4 !}+\cdots} \\
& =t^{2}+\frac{t^{2}\left(1-t^{2}\right)}{12} \alpha^{2}+O\left(\alpha^{4}\right) \\
\frac{1-\cosh (\alpha t)}{1-\cosh \alpha} & =t^{2}-\frac{t^{2}\left(1-t^{2}\right)}{12} \alpha^{2}+O\left(\alpha^{4}\right)
\end{aligned}
$$

we have

$$
Z_{2}^{*}(\alpha, t)= \begin{cases}t^{2}+\frac{t^{2}\left(1-t^{2}\right)}{12} \alpha^{2}+\cdots & (-\pi<\alpha<0) \\ t^{2} & (\alpha=0) \\ t^{2}-\frac{t^{2}\left(1-t^{2}\right)}{12} \alpha^{2}+\cdots & (\alpha>0) .\end{cases}
$$

Thus $Z_{2}^{*}(\alpha, t)$ is continuous and differentiable at $\alpha=0$. Since $D_{\alpha} Z_{2}^{*}(0, t)=\lim _{\alpha \rightarrow 0} \frac{Z_{2}^{*}(\alpha, t)}{\alpha}=0, Z_{2}^{*}\left(\alpha, t_{0}\right)$, $\alpha \in(-\pi, \infty)$ is $C^{1}$ continuous, and so are $Z_{1}^{*}(\alpha, t)$ and $Z_{0}^{*}(\alpha, t)$ by Definition 2.4. Hence $q^{*}\left(\alpha, t_{0}\right), \alpha \in(-\pi, \infty)$ is $C^{1}$ continuous.

Proposition 3.7 For each $t_{0} \in(0,1)$, the isoparametric curve $q^{*}\left(\alpha, t_{0}\right), \alpha \in(-\pi, \infty)$ is not twice differentiable.

proof. Since

$$
\begin{aligned}
& \lim _{\alpha \rightarrow 0+} \frac{D_{\alpha} Z_{2}^{2}(\alpha, t)-D_{\alpha} Z_{2}^{2}(0, t)}{\alpha}=-\frac{t^{2}\left(1-t^{2}\right)}{6} \\
& \lim _{\alpha \rightarrow 0-} \frac{D_{\alpha} Z_{2}^{2}(\alpha, t)-D_{\alpha} Z_{2}^{2}(0, t)}{\alpha}=\frac{t^{2}\left(1-t^{2}\right)}{6},
\end{aligned}
$$

the second derivative of $Z_{2}^{2}(\alpha, t)$ with respect to $\alpha$ does not exist. Thus the isoparametric curve $q^{*}\left(\alpha, t_{0}\right)$, $\alpha \in(-\pi, \infty)$ is not twice differentiable.

Remark. Even if the isoparametric curve $q^{*}(\alpha, 1 / 2)$ is a straight line segment $\overline{m q_{1}}$, it not twice differentiable.

\section{Conclusion}

In this paper, we found some geometric properties of the isoparametric curve of quadratic F-Bézier curves. We showed that the isoparametric curve is tangent to the control polygon at the end point of the curve, or a straight line, and proved that the isoparametric curves are $C^{1}$ continuous but not twice differentiable.

In future works, we have plans to find the necessary and sufficient condition for the cubic F-Bézier curve to be a conic section, and to obtain some geometric properties of the isoparametric curve of cubic F-Bézier curves.

\section{Acknowledgements}

This study was supported by research funds from Chosun University, 2012.

\section{References}

[1] H. Pottmann, "The geometry of Tchebycheffian spines", Comput. Aided Geom. D., Vol. 10, pp. 181-210, 1993.

[2] J. Zhang, "C-curves: An extension of cubic curves", Comput. Aided Geom. D., Vol. 13, pp. 199-217, 1996.

[3] J. Zhang, "Two different forms of C-B-splines", Comput. Aided Geom. D., Vol. 14, pp. 31-41, 1997,

[4] J. W. Zhang, "C-Bézier curves and surfaces”, Graph. Models Image Process, Vol. 61, pp. 2-15, 1999.

[5] E. Mainar, J. Pena, and J. Sanchez-Reyes, "Shape preseving alternatives to the raional Bézier model", Comput. Aided Geom. D., Vol. 18, pp. 37-60, 2001.

[6] Q. Chen and G. Wang, "A class of Bézier-like curves", Comput. Aided Geom. D., Vol. 20, pp. 29-39, 2003.

[7] Y. Lu, G. Wang, and X. Yang, "Uniform hyperbolic 
polynomial B-spline curves", Comput. Aided Geom. D., Vol. 19, pp. 379-393, 2002.

[8] J. W. Zhang and F.-L. Krause, "Extend cubic uniform B-splines by unified trigonometric and hyberolic basis", Graph. Models, Vol. 67, pp. 100-119, 2005.

[9] J. Zhang, F.-L. Krause, and H. Zhang, "Unifying $\mathrm{C}$-curves and $\mathrm{H}$-curves by extending the calculation to complex numbers", Comput. Aided Geom. D., Vol. 22, pp. 865-883, 2005.
[10] G. Farin, "Curves and surfaces for Computer Aided Geometric Design: A particle code", fifth ed. Academic Press, London, 2001.

[11] M. Floater, "An $O\left(h^{2 n}\right)$ Hermite approximation for conic sections", Comput. Aided Geom. D., Vol. 14, pp. 135-151, 1997.

[12] H. Y. Park, "Properties of isoparametric curve of quadratic F-Bezier curve", M.S. thesis, Chosun University, 2012. 